\title{
Proton acceleration by radially polarized chirped laser pulses
}

\author{
Jin-Lu Liu (刘晋陆), ${ }^{1}$ Z. M. Sheng (盛政明), ${ }^{1,2, *}$ J. Zheng (郑君), ${ }^{1}$ C. S. Liu (刘全生), ${ }^{3}$ and J. Zhang (张杰) ${ }^{1,2}$ \\ ${ }^{1}$ Key Laboratory for Laser Plasmas (Ministry of Education) and Department of Physics, Shanghai Jiao Tong University, \\ Shanghai 200240, China \\ ${ }^{2}$ Beijing National Laboratory of Condensed Matter Physics, Institute of Physics, CAS, Beijing 100190, China \\ ${ }^{3}$ East-West Space Science Center, University of Maryland, College Park, Maryland 20742, USA
}

(Received 27 October 2011; published 11 April 2012)

\begin{abstract}
Within the framework of plane-wave angular spectrum analysis of electromagnetic fields, a solution for the field of a tightly focused radially polarized (RP) chirped laser pulse is presented. With this solution, direct laser acceleration of protons by this kind of RP laser pulses is investigated numerically. It is found that a RP laser pulse with proper negative frequency chirps can lead to efficient proton acceleration, reaching sub-GeV at the laser intensity of $10^{22} \mathrm{~W} / \mathrm{cm}^{2}$ from its injection energy of $45 \mathrm{MeV}$.
\end{abstract}

DOI: 10.1103/PhysRevSTAB.15.041301

PACS numbers: 41.75.Jv, 42.25.Ja, 45.50.Jf

\section{INTRODUCTION}

High-energy protons and heavier ions are widely used in many areas, such as proton cancer therapy [1], proton imaging [2], ion lithography [3], and fast-ion ignition fusion research [4], etc. With the development of high power lasers, a few schemes have been proposed to obtain high-energy proton beams based upon relativistic laserplasma interaction, such as target normal sheath acceleration $[5,6]$, and radiation pressure dominated acceleration [7-9]. In these schemes, protons are accelerated by electrostatic fields arising from the charge separation between protons and the laser-pushed electrons. During the acceleration process, the development of Rayleigh-Taylor or Weibel-like instability [10] may abate proton acceleration at a later time [11-13]. On the other hand, it is well known that charged particles may be accelerated directly by intense lasers. However, due to the small charge-to-mass ratio for protons compared to electrons, usually it cannot be accelerated directly by lasers. Simple estimation shows that the kinetic oscillation energy of a proton approaches $M_{p} c^{2}$ only when laser intensity is up to $10^{24} \mathrm{~W} / \mathrm{cm}^{2}$, where $M_{p}$ is the rest mass of a proton.

Recently, it is proposed to accelerate charged particles especially electrons in vacuum by radially polarized (RP) intense laser beams. This kind of laser beams can accelerate electrons along the beam axis by its strong longitudinal electric field and meanwhile confine them by its transverse electric and magnetic fields [14-18]. A RP laser pulse can be focused to the order of a laser wavelength, which significantly increases the longitudinal electric field at focus $[19,20]$. Furthermore, it is found that a frequency

\footnotetext{
*zmsheng@sjtu.edu.cn
}

Published by the American Physical Society under the terms of the Creative Commons Attribution 3.0 License. Further distribution of this work must maintain attribution to the author(s) and the published article's title, journal citation, and DOI. chirp may contribute to the synchronization between the accelerating laser field and the accelerated electrons to prolong the acceleration distance [21,22]. In most existing work, paraxial solutions for the RP laser fields have been used and the pulse duration effect has been treated simply by multiplying a temporal profile, which are usually not accurate enough to study laser acceleration at extremely high intensity.

In this paper, a new solution for tightly focused chirped RP laser pulse is presented based on plane-wave angular spectrum analysis (ASA) $[17,18,23,24]$ and the Taylor expansion [16,23-25]. Using our new solution, we investigate proton acceleration by this kind of RP laser pulses with peak intensity around $10^{22} \mathrm{~W} / \mathrm{cm}^{2}$. It is found that a RP laser pulse with proper negative frequency chirps may lead to efficient proton acceleration.

\section{RADIALLY POLARIZED CHIRPED LASER PULSES}

We start by calculating the field structure of a tightly focused chirped RP laser pulse. It is well known that the laser pulse fields can be expressed generally by [16,23-25]

$$
\begin{aligned}
F(r, \theta, z, t)= & \int_{-\infty}^{+\infty} \tilde{f}\left[\omega(\xi)-\omega_{0}(\xi)\right] \hat{F}[r, \theta, z, \omega(\xi)] \\
& \times \exp [i \omega(\xi) \xi] d[\omega(\xi)]
\end{aligned}
$$

where $r, z$, and $\theta$ denotes radial, axial, and azimuthal coordinates in a cylindrical coordinate system, respectively, $\tilde{f}\left[\omega(\xi)-\omega_{0}(\xi)\right]$ denotes the normalized spectral distribution function and $\xi=z / c-t$, and $\omega_{0}$ is the central frequency, assuming the laser propagates along the $z$ axis. For the case of a RP laser pulse, the variable $\hat{F}[r, \theta, z, \omega(\xi)]$ representing the field components $\hat{E}_{r}, \hat{E}_{z}$, and $\hat{B}_{\theta}$ are given in ASA representation as $[17,18,23]$ 


$$
\begin{gathered}
\hat{E}_{r}[r, \theta, z, \omega(\xi)]=2 \pi i \int_{0}^{1} b[\omega(\xi), \rho] \sqrt{1-\rho^{2}} J_{1}\left(\frac{\omega(\xi)}{c} r \rho\right) \exp \left[i \frac{\omega(\xi)}{c} z\left(\sqrt{1-\rho^{2}}-1\right)\right] \rho d \rho, \\
\hat{E}_{z}[r, \theta, z, \omega(\xi)]=-2 \pi \int_{0}^{1} b[\omega(\xi), \rho] \rho J_{0}\left(\frac{\omega(\xi)}{c} r \rho\right) \exp \left[i \frac{\omega(\xi)}{c} z\left(\sqrt{1-\rho^{2}}-1\right)\right] \rho d \rho, \\
\hat{B}_{\theta}[r, \theta, z, \omega(\xi)]=2 \pi i \int_{0}^{1} b[\omega(\xi), \rho] J_{1}\left(\frac{\omega(\xi)}{c} r \rho\right) \exp \left[i \frac{\omega(\xi)}{c} z\left(\sqrt{1-\rho^{2}}-1\right)\right] \rho d \rho,
\end{gathered}
$$

where $b[\omega(\xi), \rho]=A(\xi) \rho \exp \left[-\rho^{2} / 4 \sigma(\xi)^{2}\right] L_{n}^{1}\left[\rho^{2} / 2 \sigma(\xi)^{2}\right] /$ $\sqrt{1-\rho^{2}}, A(\xi)=(-1)^{n+1} \sqrt{2} i E_{0} /\left[8 \pi \sigma(\xi)^{3}\right]$, and $\sigma(\xi)=$ $1 /\left[k(\xi) w_{0}\right]=c /\left[\omega(\xi) w_{0}\right]$. Here $L_{n}^{1}$ is the associated Laguerre polynomial of the radial mode number $n$ and the angular mode number 1 (we set $n=0$ throughout this paper in the following), $J_{0}$ and $J_{1}$ refer to the Bessel function of the first kind of order 0 and 1, respectively, $E_{0}$ is an arbitrary amplitude constant, $\rho$ is the planar polar coordinates related to the Cartesian Fourier transform as defined in
Refs. $[17,18,23], w_{0}$ is the beam waist radius, and here we have considered that the waist spot size is the same for all spectral components of the pulse. Also $\omega(\xi)=\omega \pm g \xi$ is the linearly chirped frequency [22,26,27], where $\omega$ is the frequency at $\xi=0, g=C \omega^{2}$, and $C$ is the chirp parameter. Assuming that $\hat{F}[r, \theta, z, \omega(\xi)]$ are slowly varying functions of $\omega(\xi)$ with respect to the $e^{-i \omega(\xi) t}$ term, then $\tilde{f}[\omega(\xi)-$ $\left.\omega_{0}(\xi)\right]$ is sharply peaked around the center frequency $\omega_{0}(\xi)$. One can evaluate Eq. (1) using the Taylor expansion $[16,25]$

$$
\begin{aligned}
F(r, \theta, z, t)= & \left.\sum_{n=0} \frac{1}{n !} \frac{\partial^{n} \hat{F}[r, \theta, z, \omega(\xi)]}{\partial \omega(\xi)^{n}}\right|_{\omega(\xi)=\omega_{0}(\xi)} \exp \left[i \omega_{0}(\xi) \xi\right] \int_{-\infty}^{+\infty} \tilde{f}\left[\omega(\xi)-\omega_{0}(\xi)\right]\left[\omega(\xi)-\omega_{0}(\xi)\right]^{n} \\
& \times \exp \left\{i\left[\omega(\xi)-\omega_{0}(\xi)\right] \xi\right\} d[\omega(\xi)] .
\end{aligned}
$$

If adopting a Gaussian spectral distribution $\tilde{f}\left[\omega(\xi)-\omega_{0}(\xi)\right]=t_{0} \exp \left\{-\left[\omega(\xi)-\omega_{0}(\xi)\right]^{2} t_{0}^{2} / 2\right\} / \sqrt{2 \pi}$, where $t_{0}$ is the laser pulse duration, then one can obtain the second-order temporal corrected fields

$$
\begin{aligned}
F(r, \theta, z, t)= & \hat{F}\left[r, \theta, z, \omega_{0}(\xi)\right] \exp \left[-i \omega_{0}(\xi) \xi\right] \exp \left(-\xi^{2} / 2 t_{0}^{2}\right)+\left.\frac{\partial \hat{F}[r, \theta, z, \omega(\xi)]}{\partial \omega(\xi)}\right|_{\omega(\xi)=\omega_{0}(\xi)} \exp \left[-i \omega_{0}(\xi) \xi\right]\left(-i \xi / t_{0}^{2}\right) \\
& \times \exp \left(-\xi^{2} / 2 t_{0}^{2}\right)+\left.\frac{1}{2} \frac{\partial^{2} \hat{F}[r, \theta, z, \omega(\xi)]}{\partial \omega(\xi)^{2}}\right|_{\omega(\xi)=\omega_{0}(\xi)} \exp \left[-i \omega_{0}(\xi) \xi\right]\left(1 / t_{0}^{2}\right)\left(1-\xi^{2} / t_{0}^{2}\right) \exp \left(-\xi^{2} / 2 t_{0}^{2}\right)+\cdots
\end{aligned}
$$

Notice that the $n$th term in Eq. (6) scales as $\varepsilon^{n}=1 /\left(\omega_{0} t_{0}\right)^{n}$, and $\varepsilon=1 /\left(\omega_{0} t_{0}\right)$ is a small quantity as long as $t_{0} \gg 1 / \omega_{0}$. With the help of

$$
\begin{aligned}
\frac{\partial b[\omega(\xi), \rho]}{\partial \omega(\xi)}= & \frac{(-1)^{n+1} \sqrt{2} i E_{0}}{8 \pi c^{3}} \frac{\rho}{\sqrt{1-\rho^{2}}}\left(3 \omega(\xi)^{2} w_{0}^{3}-\frac{\rho^{2}}{2 c^{2}} \omega(\xi)^{4} w_{0}^{5}\right) \exp \left(-\frac{\rho^{2}}{4 \sigma(\xi)^{2}}\right), \\
\frac{\partial^{2} b[\omega(\xi), \rho]}{\partial \omega(\xi)^{2}} & =\frac{(-1)^{n+1} \sqrt{2} i E_{0}}{8 \pi c^{3}} \frac{\rho}{\sqrt{1-\rho^{2}}}\left(6 \omega(\xi) w_{0}^{3}-\frac{7 \rho^{2}}{2 c^{2}} \omega(\xi)^{3} w_{0}^{5}+\frac{\rho^{4}}{4 c^{4}} \omega(\xi)^{5} w_{0}^{7}\right) \exp \left(-\frac{\rho^{2}}{4 \sigma(\xi)^{2}}\right),
\end{aligned}
$$

one can obtain the second-order temporally corrected fields as

$$
\begin{gathered}
E_{r}(r, \theta, z, t)=2 \pi i\left[\int_{0}^{1} \sqrt{1-\rho^{2}} b(\omega, \rho) J_{1}(Q) \exp (T) \rho d \rho+\int_{0}^{1} \sqrt{1-\rho^{2}} G_{1} \exp (T) \rho d \rho\left(-i \xi / t_{0}^{2}\right)\right. \\
\left.+\frac{1}{2} \int_{0}^{1} \sqrt{1-\rho^{2}} G_{2} \exp (T) \rho d \rho \times\left(1 / t_{0}^{2}\right)\left(1-\xi^{2} / t_{0}^{2}\right)\right] \exp \left(-\xi^{2} / 2 t_{0}^{2}\right), \\
E_{z}(r, \theta, z, t)=-2 \pi\left[\int_{0}^{1} \rho b(\omega, \rho) J_{0}(Q) \exp (T) \rho d \rho+\int_{0}^{1} \rho G_{3} \exp (T) \rho d \rho\left(-i \xi / t_{0}^{2}\right)\right. \\
\left.+\frac{1}{2} \int_{0}^{1} \rho G_{4} \exp (T) \rho d \rho \times\left(1 / t_{0}^{2}\right)\left(1-\xi^{2} / t_{0}^{2}\right)\right] \exp \left(-\xi^{2} / 2 t_{0}^{2}\right),
\end{gathered}
$$




$$
\begin{aligned}
B_{\theta}(r, \theta, z, t)= & 2 \pi i\left[\int_{0}^{1} b(\omega, \rho) J_{1}(Q) \exp (T) \rho d \rho+\int_{0}^{1} G_{1} \exp (T) \rho d \rho\left(-i \xi / t_{0}^{2}\right)\right. \\
& \left.+\frac{1}{2} \int_{0}^{1} G_{2} \exp (T) \rho d \rho \times\left(1 / t_{0}^{2}\right)\left(1-\xi^{2} / t_{0}^{2}\right)\right] \exp \left(-\xi^{2} / 2 t_{0}^{2}\right)
\end{aligned}
$$

where the three terms in each equation above represent the zeroth order, the first order, and the second order of the Taylor expansion, and variables $G_{1}, G_{2}, G_{3}, G_{4}, Q, T$, and $R$ are given by

$$
\begin{aligned}
& G_{1}=\frac{\partial b[\omega(\xi), \rho]}{\partial \omega(\xi)} J_{1}(Q)+b[\omega(\xi), \rho]\left[\frac{\partial J_{1}(Q)}{\partial \omega(\xi)}+J_{1}(Q) R\right] \\
& G_{2}=\frac{\partial^{2} b[\omega(\xi), \rho]}{\partial \omega(\xi)^{2}} J_{1}(Q)+2 \frac{\partial b[\omega(\xi), \rho]}{\partial \omega(\xi)} \frac{\partial J_{1}(Q)}{\partial \omega(\xi)}+2 J_{1}(Q) \frac{\partial b[\omega(\xi), \rho]}{\partial \omega(\xi)} R+b[\omega(\xi), \rho]\left[\frac{\partial^{2} J_{1}(Q)}{\partial \omega(\xi)^{2}}+2 \frac{\partial J_{1}(Q)}{\partial \omega(\xi)} R+J_{1}(Q) R^{2}\right], \\
& G_{3}=\frac{\partial b[\omega(\xi), \rho]}{\partial \omega(\xi)} J_{0}(Q)+b[\omega(\xi), \rho]\left[\frac{\partial J_{0}(Q)}{\partial \omega(\xi)}+J_{0}(Q) R\right], \\
& G_{4}=\frac{\partial^{2} b[\omega(\xi), \rho]}{\partial \omega(\xi)^{2}} J_{0}(Q)+2 \frac{\partial b[\omega(\xi), \rho]}{\partial \omega(\xi)} \frac{\partial J_{0}(Q)}{\partial \omega(\xi)}+2 J_{0}(Q) \frac{\partial b[\omega(\xi), \rho]}{\partial \omega(\xi)} R+b[\omega(\xi), \rho]\left[\frac{\partial^{2} J_{0}(Q)}{\partial \omega(\xi)^{2}}+2 \frac{\partial J_{0}(Q)}{\partial \omega(\xi)} R+J_{0}(Q) R^{2}\right],
\end{aligned}
$$

with $\quad \omega(\xi)=\omega_{0}(\xi), \quad Q=\frac{\omega_{0}(\xi)}{c} r \rho, \quad T=i \omega_{0}(\xi) \times$ $\left(\frac{z}{c} \sqrt{1-\rho^{2}}-t\right)+\phi 0, \quad R=i \frac{z}{c}\left(\sqrt{1-\rho^{2}}-1\right)$. Notice that the frequency chirp in the field phase is represented through the variable $T$. The variables $G_{1}, G_{2}, G_{3}, G_{4}, Q$, and $R$ are introduced to simplify the writing and they do not have real physical meanings.

Figure 1 shows the normalized axial electric field $E_{z} / E_{0}$ along the $z$ axis (i.e., $r=0$ ) at time $t=0, t=5 T_{0}, t=$ $10 T_{0}$, and $t=13 T_{0}$. The laser parameters are with laser waist radius $w_{0}=1.5 \lambda_{0}$, the laser pulse duration $t_{0}=20 T_{0}$, the initial phase $\phi_{0}=0$, and the chirp parameter $C=0.00642$, where $\lambda_{0}=2 \pi c / \omega_{0}$ is the central wavelength and $T_{0}=2 \pi / \omega_{0}$ is the central laser period. One can see that the low frequency section of the axial field continuously moves forward along the positive direction of the $z$ axis with time and its positive value increases with time. This positive part of the low frequency section may accelerate protons, which are charged positively. The main idea of this paper is that protons can be efficiently accelerated by an intense chirped RP laser pulse in vacuum when a proper chirp is adopted. A proper chirp can greatly improve the synchronization between the accelerating laser field and the protons to prolong the acceleration distance.
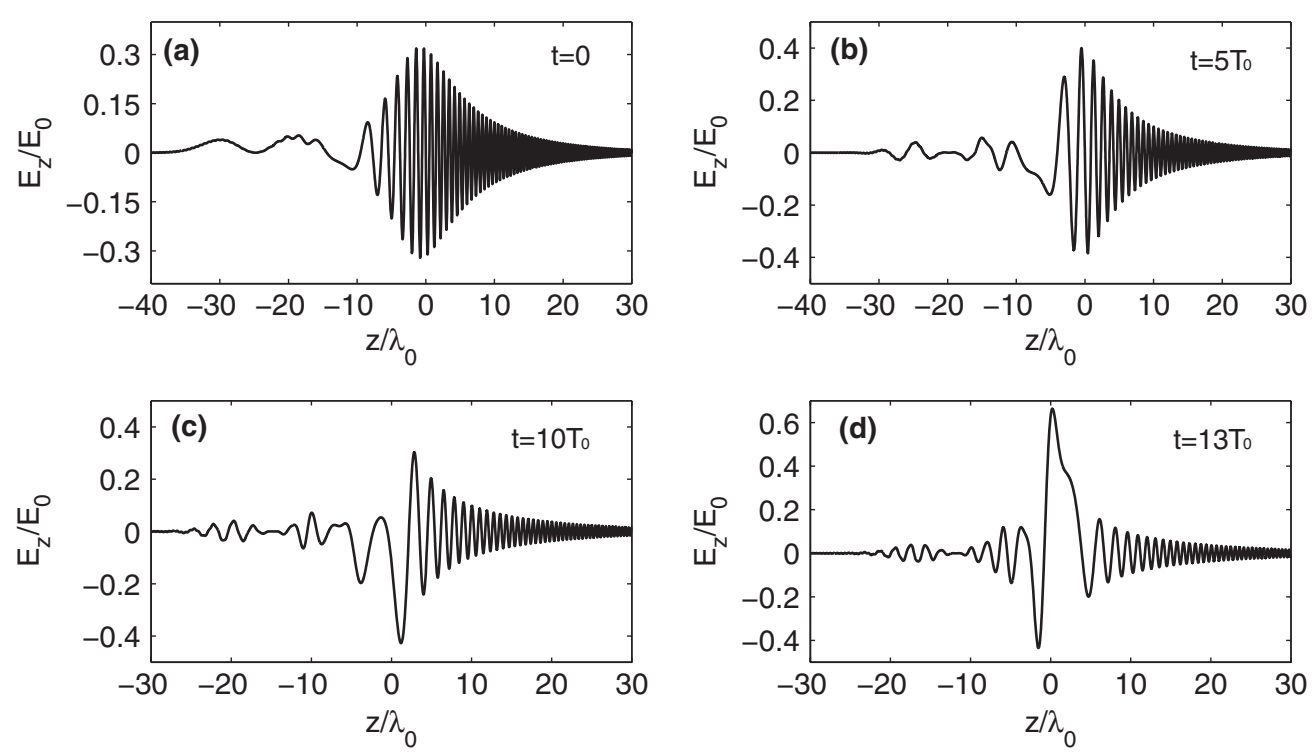

FIG. 1. The normalized axial electric field $E_{z} / E_{0}$ along the $z$ axis at different times $t=0$ (a), $t=5 T_{0}$ (b), $t=10 T_{0}$ (c), and $t=$ $13 T_{0}$ (d). The laser parameters are with the waist radius $w_{0}=1.5 \lambda_{0}$, pulse duration $t_{0}=20 T_{0}$, the initial phase $\phi_{0}=0$, and the chirp parameter $C=0.00642$. 

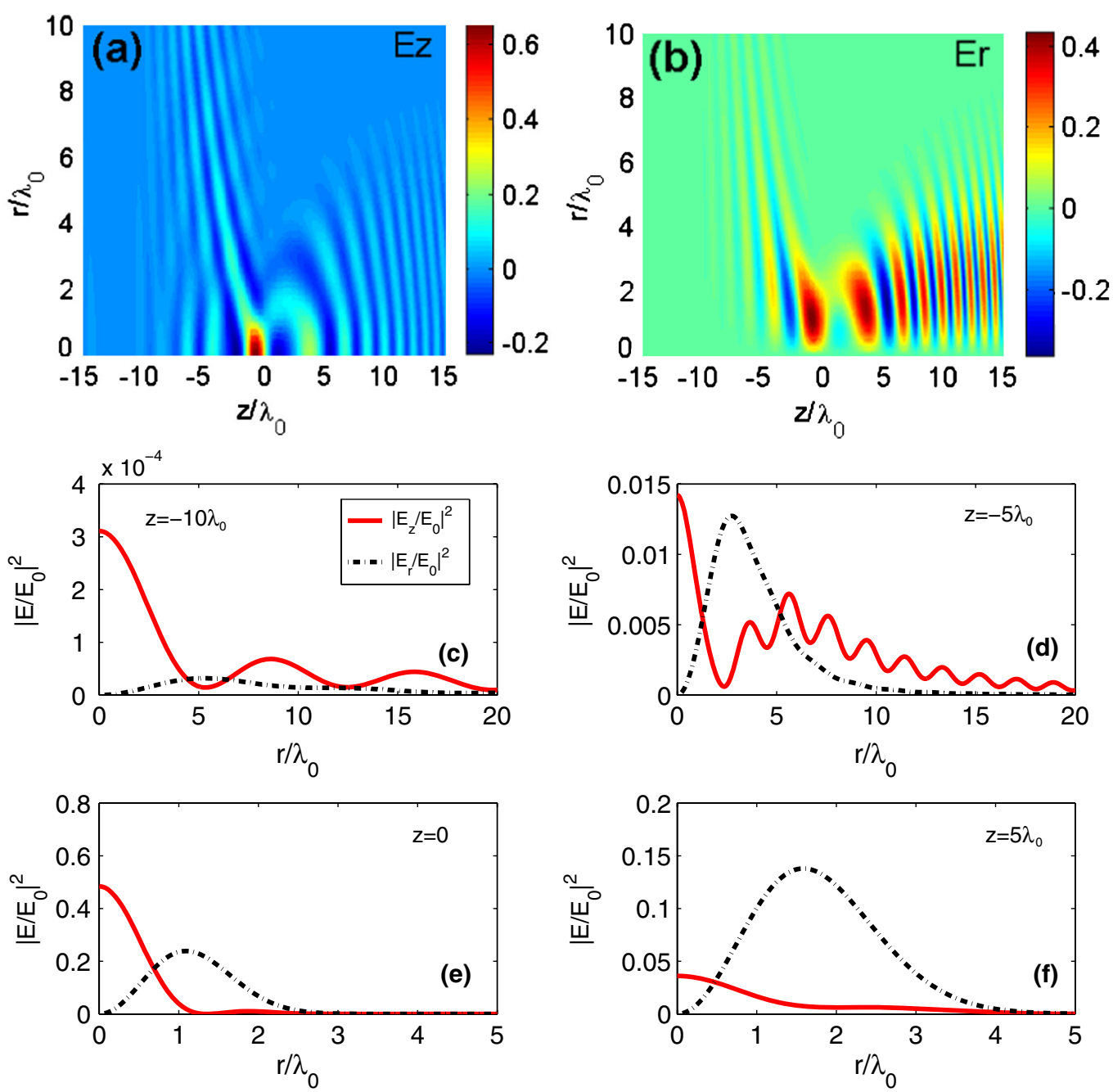

FIG. 2. The surface plots of the dimensionless amplitude of the radial field $E_{r} / E_{0}$ (a) and the axial field $E_{z} / E_{0}$ (b), respectively, on the plane $z=0$ and the radial distribution of the normalized intensity of $\left|E_{r} / E_{0}\right|^{2}$ and $\left|E_{z} / E_{0}\right|^{2}$ on different $z$ planes at $z=-10 \lambda_{0}$ (c), $z=-5 \lambda_{0}(\mathrm{~d}), z=0(\mathrm{e})$, and $z=5 \lambda_{0}$ (f). All the laser parameters are the same as in Fig. 1.

Figures 2(a) and 2(b) show the surface plots of the dimensionless amplitude of the radial field $E_{r} / E_{0}$ and the axial field $E_{z} / E_{0}$, respectively, on the $r-z$ plane at $t=$ $13 T_{0}$. Figures 2(c)-2(f) show the radial distributions of the normalized intensity of $\left|E_{r} / E_{0}\right|^{2}$ and $\left|E_{z} / E_{0}\right|^{2}$ on different $z$ planes at $z=-10 \lambda_{0}, z=-5 \lambda_{0}, z=0$, and $z=5 \lambda_{0}$. All the laser parameters are the same as in Fig. 1 . One can see that the radial field intensity exactly disappears at the $z$ axis while the axial field intensity reaches its maximum value there. One also notices that the intensity of both the radial and axial fields decay rapidly along the $r$ axis and the axial field decreases more dramatically than the radial field. The axial field always reaches its peak at the $z$ axis; meanwhile, the peak of the radial field gradually runs away from the $z$ axis as the energy of the radial field disperses along the radial direction. The above results are consistent with the basic features of the RP laser fields as discussed in previous work $[14,17,20]$. It indicates that the frequency chirp does not change the main characteristics of the radial distributions of both the radial and axial electric fields of the RP laser pulse.

\section{PROTON ACCELERATION BY RADIALLY POLARIZED LASER PULSES}

In the following, we calculate proton acceleration with the equation of motion $d \vec{p} / d t=e(\vec{E}+\vec{v} \times \vec{B} / c)$, where $e$ is the proton electric charge, $p$ and $v$ are the proton momentum and velocity, respectively, and $E$ and $B$ are the electric and magnetic fields described by Eqs. (7)-(9). In the following, length, time, velocity, momentum, proton energy, electric field strength, and magnetic field strength are normalized by $\lambda_{0}, T_{0}, c, m_{e} c, m_{e} c^{2}, m_{e} \omega_{0} c / e$, and $m_{e} \omega_{0} c / e$, respectively, where $m_{e}$ is the rest mass of electrons. The equation of motion in dimensionless form becomes

$$
d \vec{p} / d t=2 \pi(\vec{E}+\vec{p} \times \vec{B} / \gamma) / M_{p}
$$



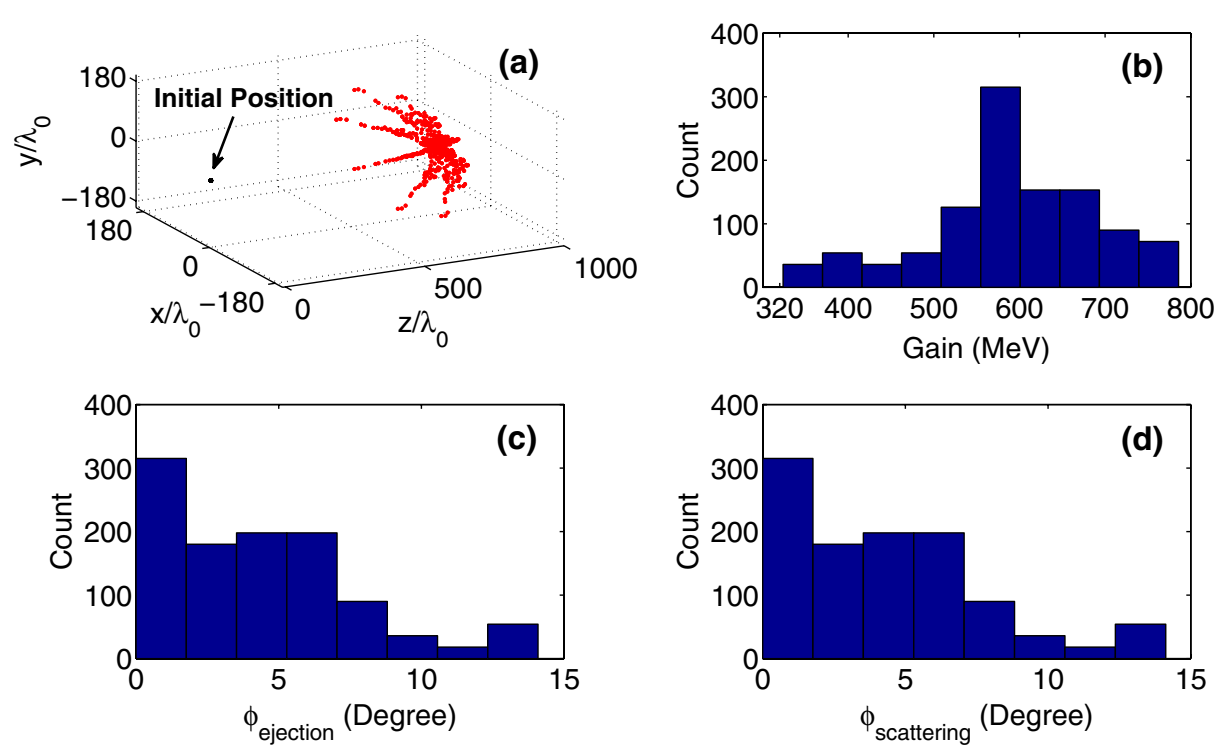

FIG. 3. Spatial distribution shown by the red dots in (a) as well as energy gain (b), ejection angle $\phi_{\text {ejection }}$ (c), and scattering angle $\phi_{\text {scattering }}$ (d) of 1000 protons initially uniformly distributed within a cylinder of length $L=0.6 \mu \mathrm{m}$ and radius $R=0.3 \mu \mathrm{m}$ [shown by the black dots in (a)] after $1000 T_{0}$. The laser pulse focus is initially located at the origin of coordinate system $(r, \theta, z)=(0,0,0)$. The center of the ensemble of protons is initially located at $(r, \theta, z)=\left(0,0,-3.7 \lambda_{0}\right)$ with the initial velocity $\left(v_{r}, v_{\theta}, v_{z}\right)=(0,0,0.3 c)$. The laser parameters are with $a_{0}=e E_{0} / m \omega_{0} c=100$, laser waist radius $w_{0}=1.5 \lambda_{0}$, the laser pulse duration $t_{0}=20 T_{0}$, the initial phase $\phi_{0}=0$, and the chirp parameter $C=0.00642$.

here we adopt $M_{p}=1836$, which is the mass ratio between proton and electron. The proton energy is determined by $d \gamma / d t=2 \pi(\vec{v} \bullet \vec{E}) / M_{p}$, where $\gamma=\sqrt{1+p_{r}^{2}+p_{z}^{2}}$.

Figure 3 plots the final spatial distribution as well as energy gain, scattering angle, and ejection angle statistics for the accelerated proton ensemble initially located near the laser focus. The proton ensemble is consisted of 1000 protons, which are initially uniformly distributed within a cylinder of length $L=0.6 \mu \mathrm{m}$ and radius $R=0.3 \mu \mathrm{m}$. The center of the ensemble of protons is initially located at $(r, \theta, z)=\left(0,0,-3.7 \lambda_{0}\right)$ with the initial velocity $\left(v_{r}, v_{\theta}, v_{z}\right)=(0,0,0.3 c)$, which corresponds to initial injection energy of $45 \mathrm{MeV}$. There are no electrons present in this proton system. The laser parameters are with $a_{0}=$ $e E_{0} / m_{e} \omega_{0} c=100$, corresponding to laser intensity $I \sim$ $1.38 \times 10^{22} \mathrm{~W} / \mathrm{cm}^{2}$, laser power $P \simeq 1.32 \mathrm{PW}$ for laser wavelength $\lambda_{0}=1 \mu \mathrm{m}$ and laser waist radius $w_{0}=$ $1.5 \lambda_{0}$. Here we take the initial phase $\phi_{0}=0$, the laser pulse duration $t_{0}=20 T_{0}$, and the chirp parameter $C=$ 0.00642 . Notice that the normalized laser field amplitude with the laser intensity mentioned above is just $a_{p}=$ $e E_{0} / M_{p} \omega_{0} c \approx 0.05$ in the light of protons with mass $M_{p}\left(=1836 m_{e}\right)$. The laser pulse focus is initially located at the origin of coordinate system $(r, \theta, z)=(0,0,0)$. We have ignored particle-particle interaction completely in calculation. The ejection angle and scattering angle are defined as $\phi_{\text {ejection }}=a \tan ^{-1}\left(p_{r f} / p_{z f}\right)$ and $\phi_{\text {scattering }}=$ $a \tan ^{-1}\left(r_{f} / z_{f}\right)$, respectively, where $p_{r f}$ and $r_{f}$ are the final radial momentum and position of protons, $p_{z f}$ and $z_{f}$ are the final axial momentum and position of protons, respectively. Figure 3(b) shows that the proton energy gain is usually larger than $300 \mathrm{MeV}$ and about $43 \%$ protons gain energy more than $600 \mathrm{MeV}$. Figures 3(c) and 3(d) show that both ejection angles and scattering angles are very small and about 59\% protons are found in angles smaller than $5^{\circ}$. This result shows that the accelerating effect is perfectly good even if the proton is placed within a limited size of space in chirped RP laser fields. As for the maximum number of protons that can be accelerated without considering collective effects, we can make a simple estimation here. First, we estimate the maximum proton density $n_{p}$ with which many-body effects can be ignored. Because $\partial E_{p} / \partial z \sim e n_{p} / \varepsilon_{0}$, one gets $E_{p} \sim e n_{p} \lambda_{0} / \varepsilon_{0}$, where $E_{p}$ is the electrostatic field between protons. Second, in our case the peak laser field $E_{L} \sim 10^{14} \mathrm{~V} / \mathrm{m}$, where $E_{L}$ is the axial electric field intensity of laser. Note that the maximum of $E_{L}$ is comparable to the radial field. If the collective field $E_{p}$ is negligible as comparable to $E_{L}, n_{p}$ should be much less than $\sim 6 \times 10^{27} \mathrm{~m}^{-3}$. Thus, taking $n_{p}=6 \times 10^{26} \mathrm{~m}^{-3}$, a radius $R=1 \mu \mathrm{m}$, and a length of $L=1 \mu \mathrm{m}$, one estimates that the maximum number of protons in the ensemble can reach $N=$ $n_{p} \pi R^{2} L \sim 2 \times 10^{9}$.

In order to ascertain the scaling relations of proton energy gain with various laser parameters, we shall only consider the acceleration mechanism of a test single proton initially located on the laser axis in the following. First, to understand why a proper negatively chirped RP laser pulse is beneficial for proton acceleration, one needs to know the 
acceleration process. Figure 4(a) shows the proton energy gain as a function of the acceleration time. It shows that significant proton energy gain occurs within the first 20 laser wavelengths. The electric field experienced by the test proton is shown in Figs. 4(b)-4(d) for three cases of negatively chirped, nonchirped, and positively chirped. Figure 4(b) shows that the proton experiences only a short period of oscillation and then a long time large positive acceleration field. However, Figs. 4(c) and 4(d) show that the proton experiences acceleration and deceleration almost symmetrically for the laser pulse either without frequency chirp or with a positive chirp, resulting in negligible energy gain. One can simply estimate the proton energy gain of these three cases from longitudinal ponderomotive force effect and the axial electric field direct acceleration effect. In the ponderomotive potential model, the time-averaged force experienced by the proton is $F_{\text {pond }}(t)=-\nabla V_{\text {pond }}(r, \theta, z, t)$, where $V_{\text {pond }}=\left[\sqrt{1+|A(r, \theta, z, t)|^{2} / 2}-1\right] M_{p} c^{2}$ and $|A(r, \theta, z, t)| / 2$ is the normalized time-averaged laser intensity profile. If
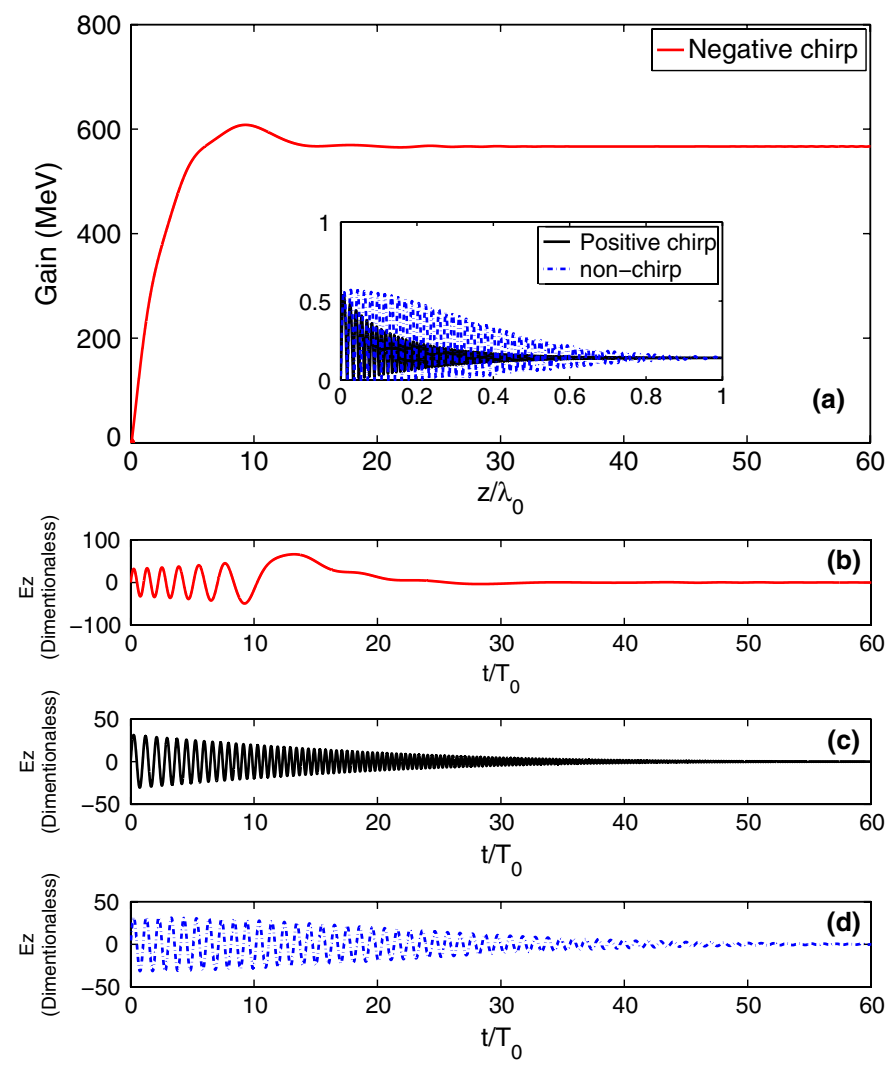

FIG. 4. (a) Energy gain of a test single proton as a function of acceleration distance for the laser pulse with different frequency chirp parameters. The initial coordinates of the test proton is $(r, \theta, z)=(0,0,0)$ and it starts from rest. The laser parameters are the same as in Fig. 3. Plots (b), (c), and (d) show the longitudinal electric fields experienced (normalized by $\left.m_{e} \omega_{0} c / e\right)$ by the test proton when the laser pulse is either negatively, positively chirped, or not chirped, respectively. simply choosing $|A(r, \theta, z, t)|$ as $a_{p} / 2 \approx 0.025$ for estimation, then one can obtain this ponderomotive potential energy as $V_{\text {pond }} \approx 0.146 \mathrm{MeV}$, which is exactly the same as the case of positively chirped and nonchirped pulses. Meanwhile, for the case of a negatively chirped pulse, the proton energy gain can be simply estimated from the timeaveraged acceleration field times the acceleration distance. If simply taking an accelerating distance $D \approx 6 \lambda_{0}$ and an average accelerating field $\bar{E} \approx 0.3 E_{0}=30 m_{e} \omega_{0} c / e$ for estimation according to Figs. 4(a) and 4(b), then one can obtain this energy gain as $G \approx 578 \mathrm{MeV}$, which is approximately the same as our numerical result.

Figures 5(a) and 5(b) show the energy gain as a function of the chirp parameter $C$ for different pulse durations. It appears that the energy gain is very sensitive to the chirp parameter. One can only choose some special values of $C$, at which the phase variation of the laser field and proton motion are matched for efficient acceleration. From the perspective of analytic analysis, the proton energy gain is $G=\left[\sqrt{1+\left(2 \pi \int_{0}^{t} E_{z} d t^{\prime}\right)^{2}}-1\right] M c^{2}$. Since the main part of $E_{z}$ is its 0th order term proportional to the term $b[\omega(\xi), \rho]=-\sqrt{2} i E_{0} \rho \exp \left[-\rho^{2} / 4 \sigma(\xi)^{2}\right] /\left[8 \pi \sigma(\xi)^{3} \times\right.$ $\left.\sqrt{1-\rho^{2}}\right]$, where $\sigma(\xi)=c /\left[\omega(\xi) w_{0}\right]$, then one can see that $E_{z} \propto \omega(\xi)^{3} \exp \left[-\omega(\xi)^{2}\right]$. Therefore a slight change in $\omega(\xi)=\omega+C \omega^{2} \xi$ can bring a considerable change in $E_{z}$ and its time integral as well as the proton energy gain. Meanwhile, the choice of $C$ should make the part of frequency variation $C \omega^{2} \xi$ smaller than $\omega$ in the range of $\xi \in\left[-t_{0}, t_{0}\right]$, otherwise the laser frequency changes its sign within the laser pulse which is unrealistic. Figures 5(c) and 5(d) show the variation of proton energy gain with the waist $w_{0}$. One notices that there exists an
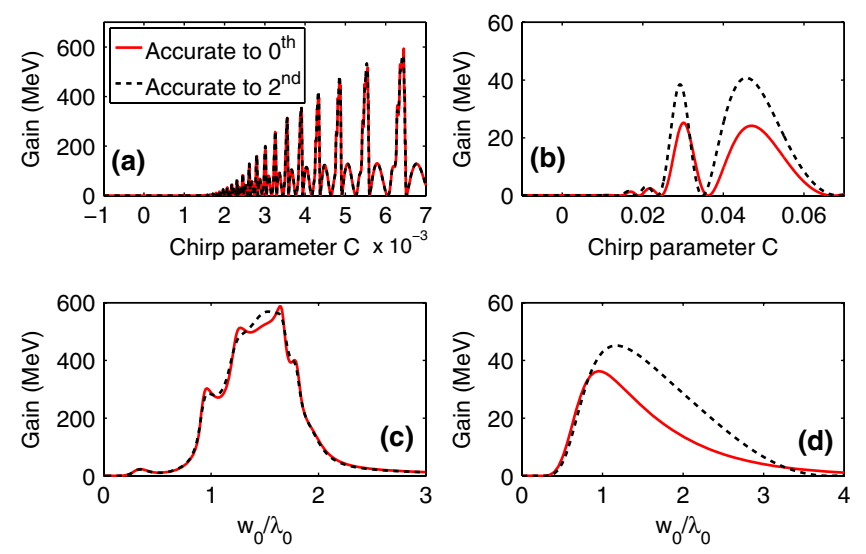

FIG. 5. Energy gain of a test single proton as a function of the chirp parameter $C$ for the pulse duration $t_{0}=20 T_{0}$ (a) and $t_{0}=$ $2 T_{0}$ (b). The energy gain of a test proton as a function of the laser beam waist $w_{0}$ is plotted in (c) and (d) for $t_{0}=20 T_{0}(C=$ $0.00642)$ and $t_{0}=2 T_{0}(C=0.046)$, respectively. The red line and the black line represent results obtained by adopting laser field accurate to 0th order and 2nd order, respectively. Other parameters are the same as in Fig. 3. 

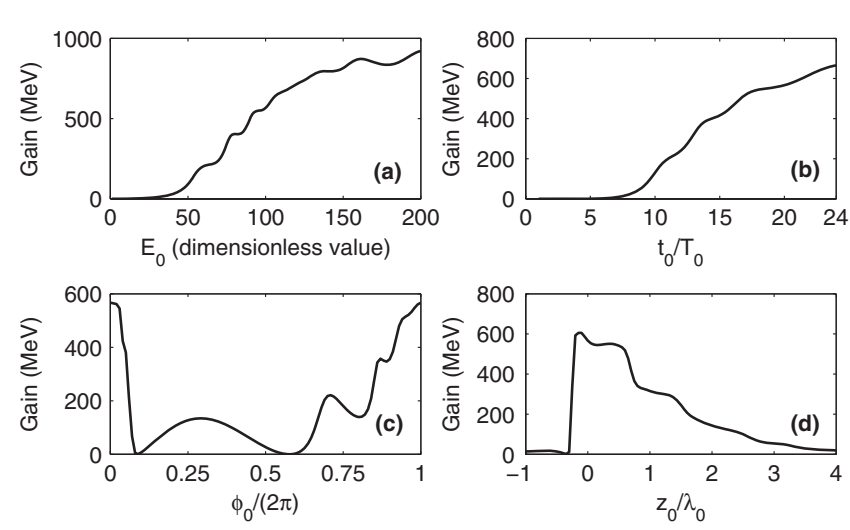

FIG. 6. Energy gain of a test single proton as a function of the peak laser amplitude $E_{0}(\mathrm{a})$, laser pulse duration $t_{0}(\mathrm{~b})$, initial phase of the laser field $\phi_{0}(\mathrm{c})$, and its initial axial position $z_{0}(\mathrm{~d})$. Other parameters are the same as in Fig. 3.

optimal value of $w_{0} \approx 1.53 \lambda_{0}$ for the case of the laser duration $t_{0}=20 T_{0}(C=0.00642)$ and $w_{0} \approx 1.15 \lambda_{0}$ for the case of the laser duration $t_{0}=2 T_{0}(C=0.046)$. This can be understood that a larger beam waist can provide a longer acceleration distance for a longer Rayleigh length, whereas a large beam waist leads to a smaller axial field for acceleration. Therefore there exists an optimal value of $w_{0}$ balancing those two competing factors to produce the maximum proton energy gain. Figures 5(a) and 5(c) show that the results for $t_{0}=20 T_{0}$ are almost the same with the field accurate to the 0th order and 2 nd order, while Figs. 5(b) and 5(d) show an apparent difference for $t_{0}=2 T_{0}$. This can be understood that the $n$th term in Eqs. (7)-(9) scales as $1 /\left(\omega_{0} t_{0}\right)^{n}$, which is negligibly small as long as $t_{0} \gg 1 / \omega_{0}$. One also notices that energy gain for $t_{0}=20 T_{0}$ is significantly large than that of $t_{0}=2 T_{0}$ when adding appropriate chirp. This can be interpreted that proper long pulse duration can prolong acceleration time as also shown in Fig. 6(b) below.

Figure 6(a) shows that the proton energy gain increases with the increase of incident peak laser amplitude $E_{0}$. Generally the proton can gain higher energy in more intense laser fields, while for $E_{0}>100$, there exists some platforms, where the proton energy changes weakly with $E_{0}$. This can be understood from the oscillating form of the longitudinal field $E_{z}$. On the one hand, with the increase of $E_{0}$, the proton longitudinal momentum is enhanced. On the other hand, the fast motion of proton easily brings a phase slip causing the proton from the acceleration phase into the deceleration phase and terminating the energy gain process. Figure 6(b) shows that properly long pulse durations can prolong acceleration time as also shown in Fig. 5. Figure 6(c) shows that the proton energy gain is sensitive to the field initial phase. The regions $0 \leq \phi_{0} \leq 0.09 \pi$ and $1.8 \pi \leq \phi_{0} \leq 2 \pi$ are proper for acceleration in negatively chirped field. Figure 6(d) shows that proton energy gain decreases rapidly with the increase of its offset from laser focus $z_{0}=0$. The change of initial axial position is equivalent to the change of the field amplitude and phase, which should result in a periodic variation. However, in the case of a chirped field, there is no periodic variation which is different from the case of a nonchirped one (not shown in this figure) because the frequency chirp breaks the periodic change of the field phase seen by the proton.

\section{CONCLUSION}

In conclusion, we have shown that protons can be efficiently accelerated by an intense radially polarized chirped laser pulse in vacuum. This is found only when a proper negative chirp is adopted for the laser pulse, which can greatly improve the synchronization between the accelerating laser field and the particles to prolong the acceleration distance. The obtained single proton energy gain can be at sub-GeV level for the peak laser intensity around $10^{22} \mathrm{~W} / \mathrm{cm}^{2}$ when the protons are injected with initial energy of $45 \mathrm{MeV}$. The accelerated protons are well collimated due to the field structure of the adopted laser pulse.

\section{ACKNOWLEDGMENTS}

This work was supported by the National Natural Science Foundation of China (Grants No. 10935002, No. 10905039, and No. 11075105) and National Basic Research Program of China (Grant No. 2009GB105002).

[1] C. M. Ma, I. Veltchev, E. Fourkal, J. S. Li, W. Luo, J. Fan, T. Lin, and A. Pollack, Laser Phys. 16, 639 (2006).

[2] M. Borghesi, A. J. Mackinnon, D. H. Campbell, D. G. Hicks, S. Kar, P. K. Patel, D. Price, L. Romagnani, A. Schiavi, and O. Willi, Phys. Rev. Lett. 92, 055003 (2004).

[3] A. Alves, P. Reichart, R. Siegele, P. N. Johnston, and D. N. Jamieson, Nucl. Instrum. Methods Phys. Res., Sect. B 249, 730 (2006).

[4] M. Roth, T.E. Cowan, M. H. Key, S. P. Hatchett, C. Brown, W. Fountain, J. Johnson, D. M. Pennington, R. A. Snavely, S.C. Wilks, K. Yasuike, H. Ruhl, P. Pegoraro, S. V. Bulanov, E. M. Campbell, M.D. Perry, and H. Powell, Phys. Rev. Lett. 86, 436 (2001).

[5] Y. Sentoku, T.E. Cowan, A. Kemp, and H. Ruhl, Phys. Plasmas 10, 2009 (2003).

[6] B. M. Hegelich, B. J. Albright, J. Cobble, K. Flippo, S. Letzring, M. Paffett, H. Ruhl, J. Schreiber, R. K. Schulze, and J. C. Fernandez, Nature (London) 439, 441 (2006).

[7] T. Esirkepov, M. Borghesi, S. V. Bulanov, G. Mourou, and T. Tajima, Phys. Rev. Lett. 92, 175003 (2004).

[8] X. Q. Yan, C. Lin, Z. M. Sheng, Z. Y. Guo, B. C. Liu, Y. R. Lu, J.X. Fang, and J.E. Chen, Phys. Rev. Lett. 100, 135003 (2008).

[9] A. Henig, S. Steinke, M. Schnürer, T. Sokollik, R. Hörlein, D. Kiefer, D. Jung, J. Schreiber, B. M. Hegelich, X. Q. Yan, J. Meyer-ter-Vehn, T. Tajima, P. V. Nickles, W. Sandner, and D. Habs, Phys. Rev. Lett. 103, 245003 (2009). 
[10] F. Pegoraro and S. V. Bulanov, Phys. Rev. Lett. 99, 065002 (2007).

[11] M. Chen, A. Pukhov, T. P. Yu, and Z. M. Sheng, Phys. Rev. Lett. 103, 024801 (2009).

[12] T. P. Yu, A. Pukhov, G. Shvets, and M. Chen, Phys. Rev. Lett. 105, 065002 (2010).

[13] B. Qiao, M. Zepf, M. Borghesi, B. Dromey, M. Geissler, A. Karmakar, and P. Gibbon, Phys. Rev. Lett. 105, 155002 (2010).

[14] Y. I. Salamin, Opt. Lett. 31, 2619 (2006).

[15] Y. I. Salamin, Z. Harman, and C.H. Keitel, Phys. Rev. Lett. 100, 155004 (2008).

[16] M. P. Liu, H. C. Wu, B.S. Xie, and M. Y. Yu, Phys. Plasmas 15, 023108 (2008).

[17] R. Martinez-Herrero, P. M. Mejias, and S. Bosch, Opt. Commun. 281, 3046 (2008).

[18] J. L. Liu, Z. M. Sheng, and J. Zheng, Opt. Commun. 284, 4646 (2011).
[19] R. Dorn, S. Quabis, and G. Leuchs, Phys. Rev. Lett. 91, 233901 (2003).

[20] Y. I. Salamin, New J. Phys. 11, 033009 (2009).

[21] K. P. Singh, Appl. Phys. Lett. 87, 254102 (2005).

[22] J. X. Li, W. P. Zang, and J. G. Tian, Appl. Phys. Lett. 96, 031103 (2010).

[23] J. L. Liu, Z. M. Sheng, and J. Zheng, Chin. Phys. B 21, 024101 (2012).

[24] B. Quesnel and P. Mora, Phys. Rev. E 58, 3719 (1998).

[25] J.F. Hua, Y. K. Ho, Y.Z. Lin, Z. Chen, Y.J. Xie, S. Y. Zhang, and J. J. Xu, Appl. Phys. Lett. 85, 3705 (2004).

[26] D. N. Gupta, H. J. Jang, and H. Suk, J. Appl. Phys. 105, 106110 (2009).

[27] B. J. Galow, Y. I. Salamin, T. V. Liseykina, Z. Harman, and C.H. Keitel, Phys. Rev. Lett. 107, 185002 (2011). 